\title{
A Case of Low Grade Thyroid Like Nasopharyngeal Papillary Adenocarcinoma in Posterior Nasal Septum
}

\author{
Chang Lok Ji, $\mathrm{MD}^{1}$, Geun Hyung Park, $\mathrm{MD}^{1}$, Soo Kweon Koo, $\mathrm{MD}, \mathrm{PhD}^{1}$, and Hwaseon Lee, $\mathrm{MD}, \mathrm{PhD}^{2}$ \\ ${ }^{l}$ Department of Otorhinolaryngology-Head and Neck Surgery; ${ }^{2}$ Pathology, Busan Saint Mary's Hospital, \\ Busan, Korea
}

\begin{abstract}
- ABSTRACT -
Nasopharyngeal papillary adenocarcinoma of low-grade malignancy (LGNPPA) is an extremely rare neoplasm. A thyroid-like LGNPPA morphologically resembles a papillary thyroid carcinoma; the lesion is usually a polypoid or pedunculated mass $<1-3 \mathrm{~cm}$ in diameter, often in the posterosuperior wall of the nasopharynx. Here, we report a case of a thyroid-like LGNPPA in the left posterior septal wall. Surgical removal of the tumor was performed by transnasal endoscopic resection using laryngeal cutting forceps. The tumor was completely resected, bleeding was minimal, and the patient had no complaints of nasal pain, nasal congestion, or epistaxis. Further evaluation including immunohistochemistry confirmed a diagnosis of thyroid-like LGNPPA. No postoperative adjuvant treatment was administered. (J Clinical Otolaryngol 2019;30:70-73)
\end{abstract}

KEY WORDS: Nasal septum · Neoplasms · Adenocarcinoma, papillary.

\section{Introduction}

Low-grade nasopharyngeal papillary adenocarcinomas (LGNPPAs) are very rare; the tumor was first described and characterized by Wenig et al. ${ }^{1)}$ in 1988. In the 2005 World Health Organization (WHO) classification of head-and-neck tumors, malignant epithelial neoplasms arising in the nasopharynx are divided into the following three entities: nasopharyngeal carcinomas, salivary gland-type carcinomas, and nasopharyngeal papillary adenocarcinomas of low-grade malignancy (LGNPPAs). The latter two tumor types are classified as adenocarcinomas or primary nasopharyngeal adenocarcinomas and are very rare, constituting only $0.7 \%$ of all malignant epithelial neoplasms

논문접수일: 2019년 12월 29일

논문수정일: 2020년 3월 12일

심사완료일: 2020년 5월 6일

교신저자: 구수권, 48575 부산광역시 남구 용호로

232번길 25-14 부산성모병원 이비인후과

전화: (051) 933-7214-전송: (051) 956-1956

E-mail: koosookweon@naver.com in this region. In the nasopharynx, salivary gland-type carcinomas arise from submucosal seromucinous glands and are of various histological types, as are carcinomas of the major and minor salivary glands. In contrast, an LGNPPA is a region-specific tumor originating in the nasopharyngeal surface epithelium. ${ }^{2)}$ Thyroid-like LGNPPAs (TL-LGNPPAs) constitute a small minority of LGNPPAs and are characterized by abnormal expression of thyroid transcription factor-1 (TTF-1), thus mimicking papillary thyroid carcinomas. A TL-LGNPPA was first described by Carrizo et al. ${ }^{3)}$ in 2005. TL-LGNPPA predominantly occurs in the roof of the nasopharynx and at the posterior edge of the nasal septum. ${ }^{4}$ In a review of TL-LGNPPAs, only 9 of 25 cases arose from the nasal septum. ${ }^{5}$ Here we describe a TL-LGNPPA arising from the posterior nasal septum in a 25 -year-old male, followed by a brief discussion. 


\section{Case Report}

A 25-year-old Korean male discovered a mass on his nasal septum and was referred to the Department of Otolaryngology, Busan Saint Mary's Hospital for further evaluation. A single, protruding solid mass about 4-5 $\mathrm{mm}$ in diameter was evident on nasopharyngoscopy of the left posterior septal wall (Fig. 1). Laboratory data were unremarkable; the C-reactive protein level was normal. Diagnostic computed tomography $(\mathrm{CT})$ revealed a $0.4 \times 0.3 \mathrm{~cm}$-sized, solid nodu-

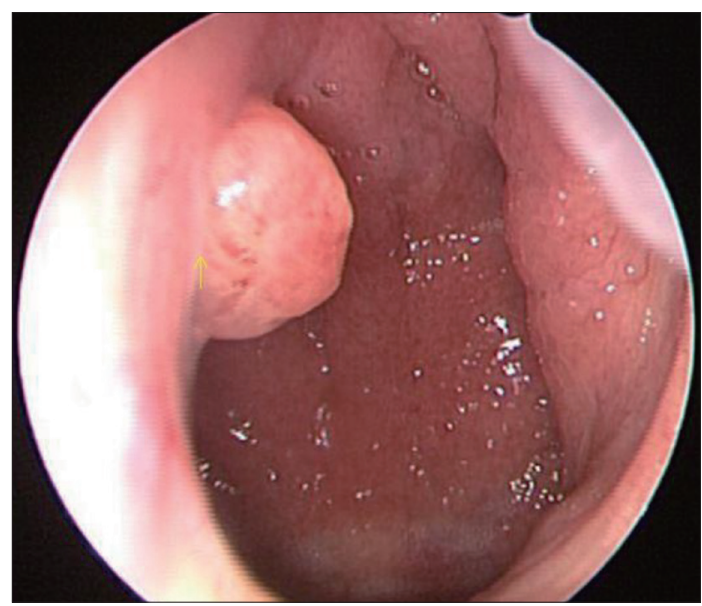

Fig. 1. Single protruding solid mass, about $45 \mathrm{~mm}$ sized on I eft posterior septal wall.
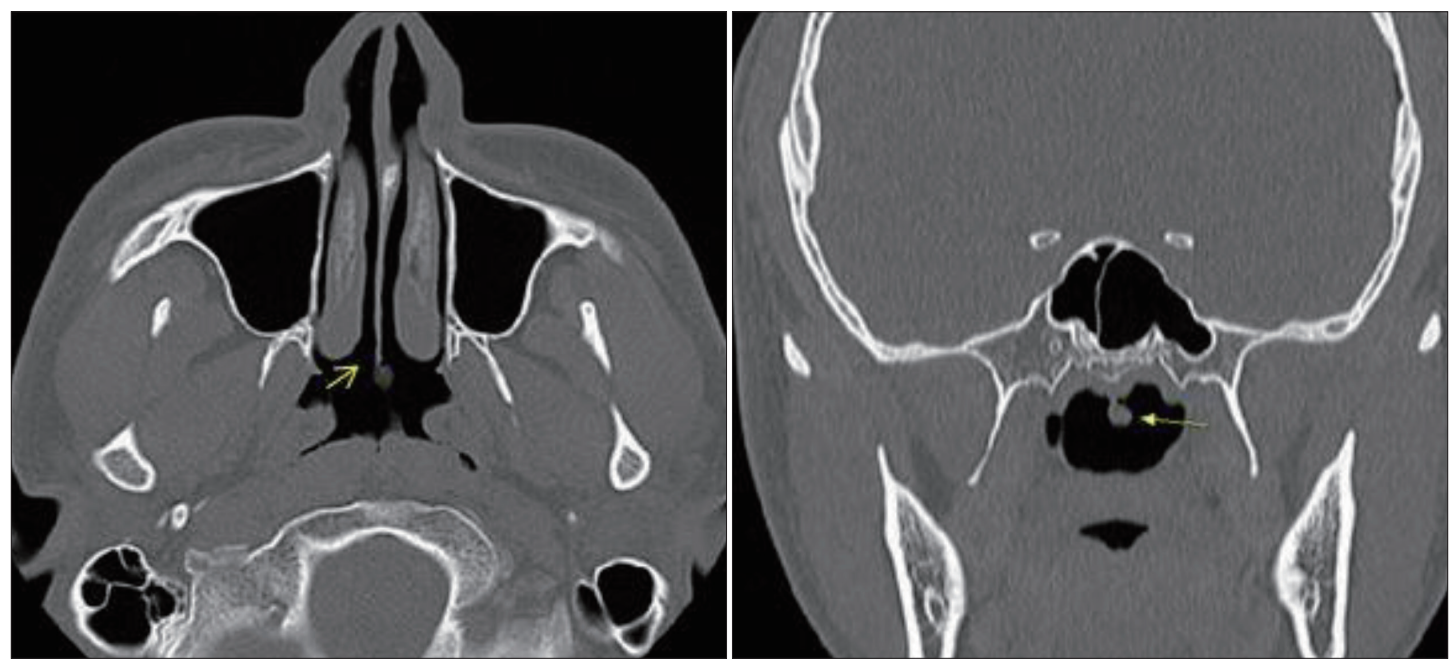

Fig. 2. Preoperative paranasal co mputed tomography (CT) images. A xial and coronal images show a $4 \times 3 \mathrm{~mm}$ sized solid nodular mass at the left posterior end of the nasal septum, lacking any adhesion to adjacent tissue. lar tumor confined to the left posterior end of the nasal septum (Fig. 2). Neither CT nor physical examination of the cervical lymph nodes revealed any abnormality. A regional excisional biopsy was performed under local anesthesia. We decided to perform transnasal endoscopic resection employing laryngeal cutting forceps to approach and resect the tumor. The tumor was completely removed, bleeding was minimal, and the patient did not complain of nasal pain, congestion or epistaxis. Microscopically, the tumor exhibited a papillary structure with a fibrovascular core. A psammoma body was evident, as in thyroid carcinomas. Most papillae were lined with epithelium, and exhibited roundto-ovoid nuclei, again similar to thyroid papillary carcinomas (Fig. 3).

On immunohistochemical examination, the tumor cells were diffusely positive for TTF-1 but negative for other thyroid-related proteins including thyroglobulin (TG). The neoplastic cells were positive for cytokeratin (CK) 7 and negative for S-100 (Fig. 3). Thus, we diagnosed a TL-LGNPPA. Thyroid function tests performed after biopsy confirmed that the patient was euthyroid. Thyroid ultrasound revealed a normal gland lacking any focal lesion or obvious jugular lymph node enlargement. We scheduled systemic radiological imaging. TORSO F18 fluorodeoxyglucose (FDG)-posi- 

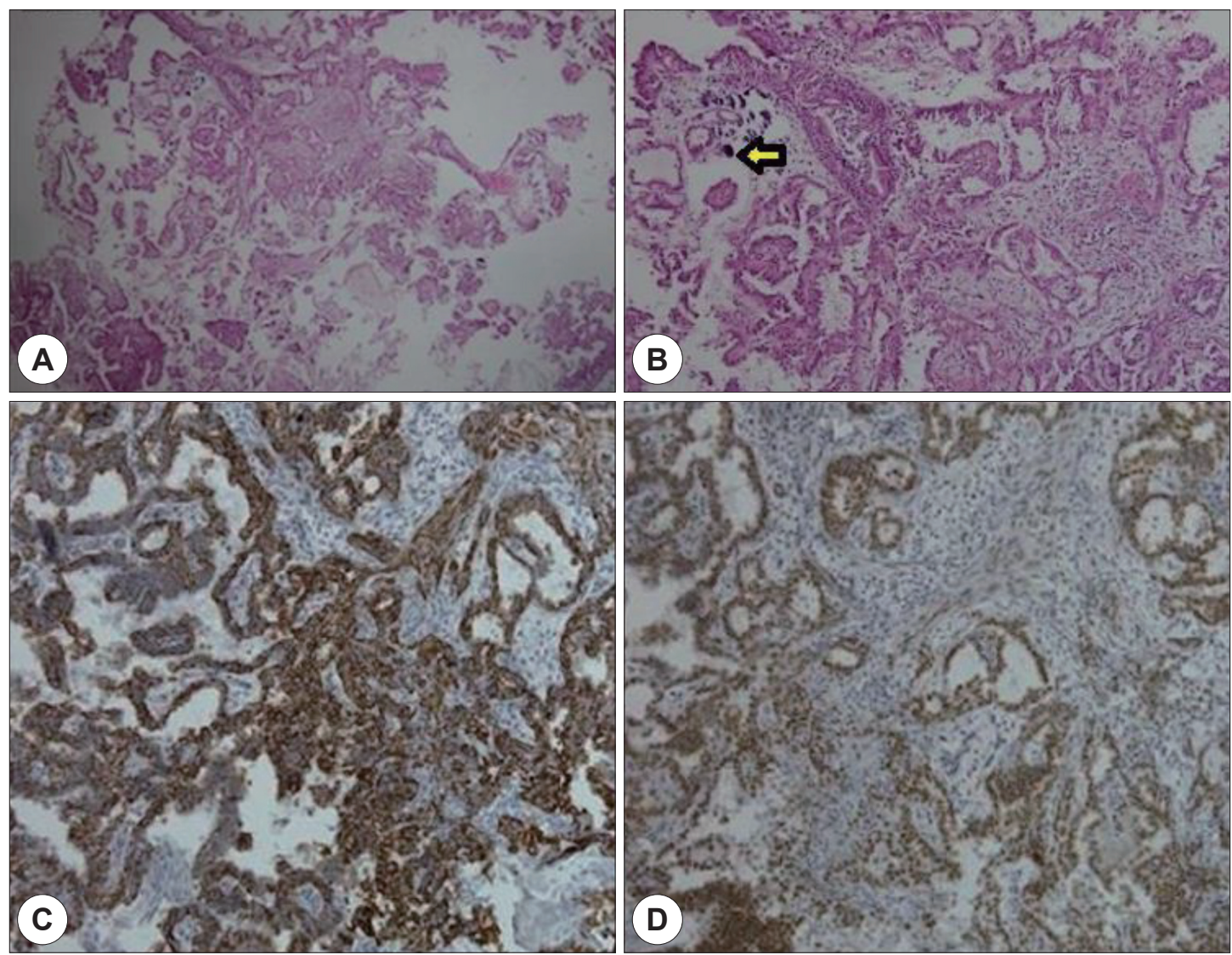

Fig. 3. Tumor exhibited a papillary structure with a fibrovascular core. Most papillae were lined with epithelium and the cells had round to ovoid nuclei, similar to thyroid papillary carcinoma cells, psammoma body was evident (arrow) (A: Hematoxylin and eosin staining, $\times 40$, B: Hematoxylin and eosin staining, $\times 100)$. Immunohistochemical staining $\times 100$. The tumor cells were CK7 (C) and TTF 1 positive (D).

tron emission tomography (PET) CT was performed $1 \mathrm{~h}$ after intravenous injection of $11.87 \mathrm{mCi}$ F18 FDG. No abnormal FDG uptake was evident from the skull base to the upper thigh; we thus confirmed the absence of a metastatic lesion. No postoperative adjuvant treatment was administered. The patient remains well and free of disease 9 months after surgery.

\section{Discussion}

LGNPPA is an extremely rare neoplasm. ${ }^{6}$ A thyroidlike LGNPPA morphologically resembles a papillary thyroid carcinoma; the nuclei are immunoreactive for TTF-1. ${ }^{7)}$ A TL-LGNPPA is a type of the principal pap- illary adenocarcinoma group of nasopharyngeal tumors. The tumor arises in the epithelium lining the nasopharynx, exhibits no gender bias, and can develop over a wide age range(11-64, mean 37 years). ${ }^{7)}$ The lesion is usually a polypoid or pedunculated mass $<1-3 \mathrm{~cm}$ in diameter, often in the posterosuperior wall of the nasopharynx. ${ }^{8}$ ) Only 9 of 25 cases of TLLGNPPAs arose from the nasal septum. ${ }^{5)}$ In this case, the mass was confined to the posterior nasal septum; a TL-LGNPPA confined to the nasal septum have not been reported so far in Korea. Differential diagnosis requires microscopic evaluation and immunohistochemical staining. As an LGNPPA is a pedunculated nodular mass $<3 \mathrm{~cm}$ in diameter, it can be misdiagnosed 
as a benign tumor such as a nasal polyp. It is difficult to distinguish an LGNPPA from a benign adenoma; pathological diagnosis is essential.

Malignant epithelial neoplasms arising in the nasopharynx are divided into the following three entities: nasopharyngeal carcinomas, salivary gland-type carcinomas, and nasopharyngeal papillary adenocarcinomas of low-grade malignancy (LGNPPA). LGNPPA and salivary gland-type carcinomas are histologically similar, ${ }^{9}$ but the latter carcinomas are surrounded by submucosal capsules; an LGNPPA exhibits a transitional zone below a normal epithelium. Immunohistochemical (IHC) staining is helpful; a salivary glandtype adenocarcinoma can be easily excluded, being S-100-negative. ${ }^{10)}$ To distinguish an LGNPPA from other nasopharyngeal carcinomas, IHC staining for TTF and EMA is required. As an LGNPPA is morphologically similar to a thyroid papillary carcinoma, IHC staining for TG is required to confirm that the tumor is not of thyroid origin. The most common IHC staining pattern of TL-LGNPPA tumor cells is TTF-1-positive TGnegative; a papillary thyroid carcinoma is TTF-1-negative TG-positive.

To the best of our knowledge, a TL-LGNPPA is a benign tumor exhibiting indolent behavior with an excellent prognosis, and thus, without any angiolymphatic spread or metastasis. The treatment of choice is complete resection. ${ }^{11)}$ If complete resection is impossible, adjuvant radiotherapy should be considered. ${ }^{1)}$ Most patients with TL-LGNPPAs report nasal symptoms such as epistaxis or nasal obstruction; there are no specific symptoms. Our patient presented because of accidental discovery of a nasal mass, and he had no specific symptoms. TL-LGNPPAs are rare. Nasopharyngeal papillary adenocarcinoma is less common than epithelial or salivary gland carcinomas, and since it shows histological findings and expression of TTF-1 similar to thyroid papillary carcinoma, it is essential to exclude the possibility of metastatic cancer. Therefore, an accurate differential diagnosis and appropriate surgical resection should afford an excellent prognosis.

\section{REFERENCES}

1) Wenig BM, Hyams VJ, Heffner DK. Nasopharyngeal papillaryadenocarcinoma. A clinicopathologic study of a lowgrade carcinoma. Am J Surg Pathol 1988;12:946-53.

2) Oide $T$, Kadosono O, Matsushima J, Wu D, Nagashima $H$, Saigusa H, et al. Thyroid-like low-grade nasopharyngeal papillary adenocarcinoma with squamous differentiation: a novel histological finding. Hum Pathol 2017;70:43-8.

3) Carrizo F, Luna MA. Thyroid transcription factor-1 expression in thyroid-like nasopharyngeal papillary adenocarcinoma: report of 2 cases. Ann Diagn Pathol 2005;9(4):188-92.

4) Oishi N, Kondo T, Nakazawa T, Mochizuki K, Kasai K, Inoue T, et al. Thyroid-like low-grade nasopharyngeal papillary adenocarcinoma: case report and literature review. Pathol Res Pract 2014;210(12):1142-5.

5) Li M, Wei J, Yao X, Wang C. Clinicopathological features of low-grade thyroid-like nasopharyngeal papillary adenocarcinoma. Cancer Res Treat 2017;49(1):213-8.

6) Chung JY, Oh DW, Kang MS, Lee JH. An unusual case of thyroid-like low grade papillary adenocarcinoma of the nasopharynx. J Clinical Otolaryngol 2017;28:283-7.

7) Sourati A, Malekzadeh M, Rakhshan A. Thyroid-like lowgrade papillary adenocarcinoma of nasopharynx, BMJ Case Rep 2019;12(6):e226949.

8) Zhang WL, Ma S, Havrilla L, Cai L, Yu CQ, Shen S, et al. Primary thyroid-like low-grade nasopharyngeal papillary adenocarcinoma: a case report and literature review. Medicine (Baltimore) 2017;96(47);e8851.

9) Sillings CN, Weathers DR, Delgaudio JM. Thyroid-like papillary adenocarcinoma of the nasopharynx: a case report in a 19-year-old male. Oral Surg Oral Med Oral Pathol Oral Radiol Endod 2010;110(3):e25-e8.

10) Pineda-Daboin K, Neto A, Ochoa-Perez V, Luna MA. Nasopharyngeal adenocarcinomas: a clinicopathologic study of 44 cases including immunohistochemical features of 18 papillary phenotypes. Ann Diagn Pathol 2006;10(4):215-21.

11) Rajeswari B, Sukumaran Nair RK, Parukuttyamma K, Mathews A. Low-grade papillary adenocarcinoma of nasopharynx with expression of thyroid transcription factor-1: case report and review of literature. Indian J Pathol Microbiol 2016;59(4): 518-20. 